\title{
Modern Software Stack Building for HEP
}

\author{
Graeme A Stewart ${ }^{1, *}$, Benjamin Morgan ${ }^{2, * *}$, Javier Cervantes Villanueva ${ }^{1,}$, and Hobbs A \\ Willett $^{3}$ \\ ${ }^{1}$ CERN, 1 Esplanade des Particules, 1211 Geneva 23, Switzerland \\ ${ }^{2}$ University of Warwick, Coventry CV4 7AL, United Kindgdom \\ ${ }^{3}$ University of Sheffield, Sheffield S10 2TN, United Kindgdom
}

\begin{abstract}
High-Energy Physics has evolved a rich set of software packages that need to work harmoniously to carry out the key software tasks needed by experiments. The problem of consistently building and deploying these packages as a coherent software stack is one that is shared across the HEP community. To that end the HEP Software Foundation Packaging Working Group has worked to identify common solutions that can be used across experiments, with an emphasis on consistent, reproducible builds and easy deployment into CernVM-FS or containers via CI systems. We based our approach on well-identified use cases and requirements from many experiments. In this paper we summarise the work of the group in the last year and how we have explored various approaches based on package managers from industry and the scientific computing community. We give details about a solution based on the Spack package manager which has been used to build the software required by the SuperNEMO and FCC experiments and trialled for a multi-experiment software stack, Key4hep. We shall discuss changes that needed to be made to Spack to satisfy all our requirements. We show how support for a build environment for software developers is provided.
\end{abstract}

\section{Introduction}

Software plays a critical role in the lifecycle of High-Energy Physics (HEP) experiments. From the high-level triggers of the experiments, through the reconstruction chain, to the identification of analysis objects, and finally to the final physics analysis, software is ubiquitous. In parallel, the simulation chain, generating physics events and simulating their passage through the detector, compliments this data-driven chain and is entirely done by software.

As HEP software has become more sophisticated so the dependencies on libraries and supporting software components have become more important. This leads to the need to build modern HEP experiment software as both a deep stack, with many dependencies building on top of one another; and as a wide stack, with several hundred packages in total performing many different functions. These packages range from entirely generic parts of a modern Linux environment, to HEP dedicated libraries, all the way up to experiment specific code. The model of a typical HEP application is shown in Figure 1.

\footnotetext{
*e-mail: graeme.andrew.stewart@ cern.ch

**e-mail: ben.morgan@warwick.ac.uk
} 


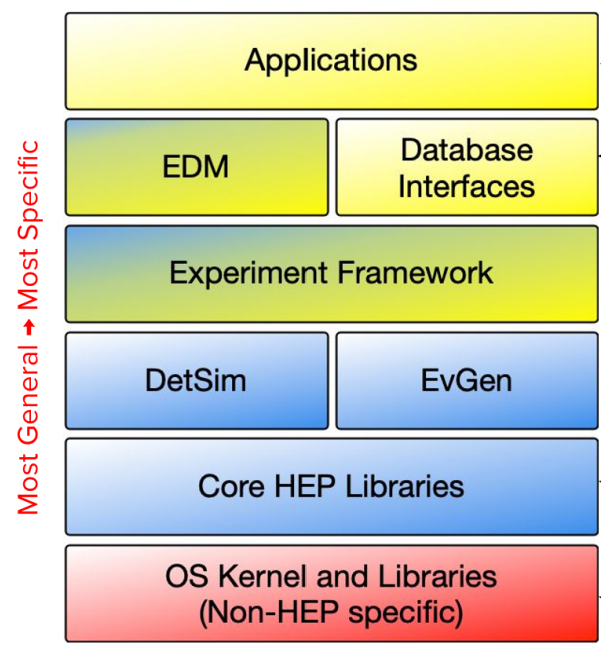

Figure 1. Illustrative figure showing a typical HEP software stack, with packages categorised from most general (bottom) to most specific (top). The lowest level components are generic to all systems, moving to scientific libraries, to common HEP packages to, finally, experiment specific software.

This deep and wide set of dependencies needs to be managed carefully, particularly for reasons of build consistency and validation (see \$2), thus HEP software should be built coherently as a software stack. As much of this work is common among experiments, the HEP Software Foundation (HSF) has had a long standing Packaging Working Group[1] that has attempted to find common solutions to this problem. In this paper we report on the recent activity of the group and, in particular, on the Spack package manager[2] which is being trialed as a possible common solution for multiple experiments.

\section{HSF Packaging Working Group}

The HSF Packaging Working Group[1] was formed in 2015 to encourage communication between the librarians of HEP experiments, who are charged with building and maintaining their experiments' software. The group focused on:

- Common build recipes and tools

- How to take most advantage of technologies like containers

- Proper support for developers and users in our collaborations

The group organised a series of meetings that reviewed packaging practice within the experiments and also looked at different tools that could be used for HEP stack building. These ranged from generic open source tools to HEP specific approaches. This early work of the group was summarised in an HSF report [3].

In 2018 the group held a series of meetings to properly define the use cases for packaging and deployment tools in HEP. This resulted in the publication of the HSF Packaging Use Cases document[4]. The main use cases identified were:

- Build

- Builds should be completely reproducible 
- Support for multiple build 'flavours' should be available (e.g. for different versions of the software or for using different compiler options)

- Toolchains and libraries should be able to be used from external sources (i.e. not under the control of the packaging tool)

- The build process should be optimised to re-use compatible binary artefacts from previous builds

- Incremental builds should be supported, where only a subset of packages are rebuilt (changed packages and their downstream dependencies)

- Build recipes should be easily shared amongst librarians and customised recipes should be easy to use, overriding defaults

- Build artefacts should be able to be stored outside of the build area itself and need to convey enough metadata to allow installation from the artefact cache without reference to the original build area

- Deployment

- Builds must be installable to different target paths, implying full install time relocatability

- Build artefacts should be convertible to 'standard' package formats, such as RPMs or deb packages

- Installations of packages built with different build options must be supported, without interfering with each other; however, it is desirable that common lower level components would only be installed once

- Removal of a release, or part of a release, must be supported

- It should be possible to update a release, should a fix need to be applied to a component

- Development

- Developers should be able to compile against any deployed release

- Developer-built packages must take precedence in the runtime development environment

- Developers can setup an environment to recompile any external or component of the software stack

These use cases can then be used when evaluating the suitability of any tool that can manage the build and install of a software stack for HEP.

\section{The Spack Package Manager}

During 2018 and 2019 the packaging working group evaluated a number of promising tools for building and installing HEP software. This work built on the work of the earlier technical note from the group[3].

One tool in particular seemed to be capable of meeting most of the requirements derived from the use cases presented above, viz. the Spack Package Manager[2], developed by Lawrence Livermore National Laboratory. This tool had come to the attention of the group shortly after the publication of HSF-TN-2016-03. Resulting interactions between HEP package managers and librarians with the Spack development team demonstrated a great willingness on their part to adapt to the specific requirements of HEP and to accept patches from our community in order to address any shortcomings that were identified.

In particular, HEP authors worked hard to improve relocation features in Spack, to develop better support for binary caches and to chain Spack instances together in so-called Spack chains. 
Our excellent working relationship with the Spack developers resulted in the addition of a dedicated HEP channel to the Spack Slack community.

\subsection{Key Spack Features}

- Unique hashes identifying a package's dependencies and build options

- Package recipes written in Python

- Hierarchy of build recipes supported

- Binary caches supported for build artefacts

- Use of system or externally pre-built packages

- Relocatable installation supported from the binary cache

- Use of RPATH to express dependencies in a robust way, ensuring that multiple versions of binaries and libraries can co-exist without interference

- Downstream instances of Spack can reuse packages installed in an upstream Spack instance (so-called Spack chains)

Given the large feature set of Spack, the significant scientific user community (much larger than HEP or even physics) and the positive engagement with the core developers, a number of HEP projects began to use the tool for their software builds.

\section{Current Use of Spack in HEP}

\subsection{Future Circular Collider}

The Future Circular Collider[5] is a next generation accelerator project proposed for CERN that would reside in a $100 \mathrm{~km}$ tunnel that could house $e e, e h$ or $h h$ colliders. To undertake detector design studies for experiments at FCC a full software stack is needed. At the time that the FCC studies began the most mature software stack available was the LCG stack, built by the CERN EP-SFT SPI project[6]. Therefore, as an expedient decision, the additional packages for FCC were built on top of existing LCG releases. This required extensive use of Spack's ability to take pre-built software, by specifying a package's source in a "packages . yaml" configuration file, e.g., to use the LCG built version of ROOT:

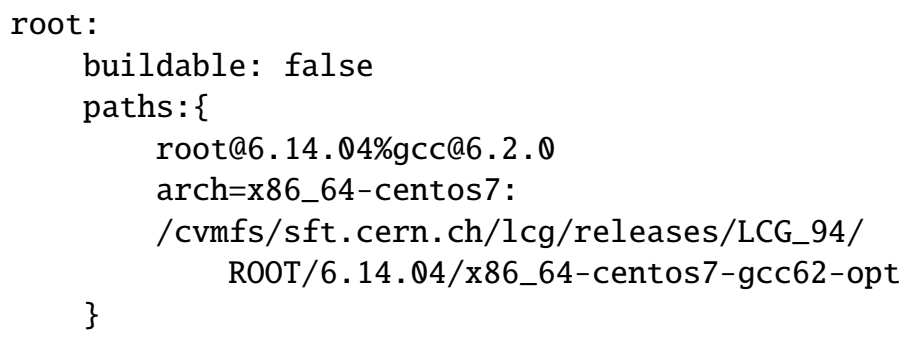

Once the LCG base is setup correctly, Spack then takes care of building the additional FCC packages, as shown in Figure 2.

Once binary artefacts have been created by Spack on the build machine, these are moved to a network filesystem that the FCC CernVM-FS[7] master has access to. On the CernVMFS master, Spack is re-run to install and relocate the packages into the final location under /cvmfs/fcc. cern. ch, where it becomes globally accessible to users. 


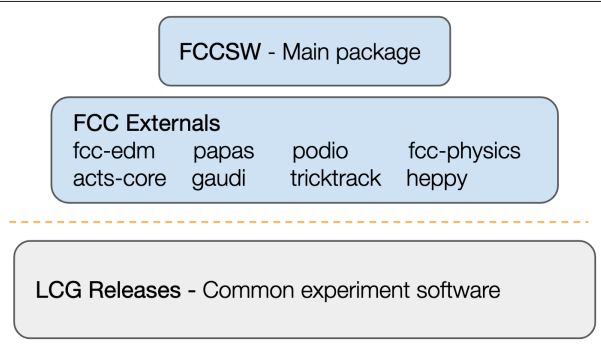

Figure 2. The FCC software stack, which is built with Spack (upper blue boxes), but bootstrapped from the CMake built LCG release (lower grey box).

One problematic issue was that re-running Spack on the CernVM-FS master effectively locked the architecture, e.g. x86_64-centos7, to that of the CernVM-FS host machine. To install for different architectures a workaround, using a Docker container with the target architecture, was used. However, recent enhancements to Spack have allowed an option for Spack to install for any specified architecture, independently of the current host, so, thanks to HEP contributors, this problem has been overcome.

Once installed in CernVM-FS, a simple shell script is provided that sets up the runtime and development environment for users.

\subsection{SuperNEMO}

SuperNEMO[8] is a Neutrinoless Double Beta Decay experiment. This is a small experiment, with little dedicated computing effort, so an off-the-shelf solution for package building and distribution is very desirable. A previous solution, based on homebrew[9] had effectively reached the limits of its capabilities.

SuperNEMO software is not currently deployed via CernVM-FS and the main build targets are native OS installation and Docker containers. A wide variety of platforms are supported: CentOS7, Ubuntu 18.04, macOS Mojave/Catalina natively; plus CentOS7 Docker/Singularity images. Similar to FCC, use is made of Spack's packages . yaml feature to reuse appropriate system binaries, which greatly reduces the build time (particularly for X11 and OpenGL packages).

Spack's hierarchical package definition features are exploited to allow for some customised versions of particular packages, such as Qt, while relying on the main Spack repository for the vast majority of packages.

At present, containers are built from scratch; the use of a binary cache to share build artefacts is under development, as is deployment to CernVM-FS

\subsection{Key4hep Prototype}

For studies of detectors beyond the HL-LHC era, there are different requirements from those at running experiments. As even accelerator parameters for ILC[10], CLIC[11], FCC[5] or CEPC [12] are not yet fixed, and there are many design options available for detectors, the need is for a software stack that is very flexible and able to rapidly be used for physics studies of different detector options. Interest in a common solution from multiple communities[13] has motivated building such a stack also using Spack as the prototype tool. 
First of all, Spack was set to bootstrap its own build of the gec compiler toolchain (on the CentOS7 platform). Binary artefacts for this toolchain were stored in a commonly accessible area for several independent Spack installations. This compiler was then used to build a baseline stack of software, that can encapsulate an HEP software workflow from event generation through to analysis. In particular Pythia8, Geant4, DD4hep, Gaudi and ROOT were built, with Spack itself being used to resolve and build all of the dependent libraries and packages, which finally numbered over 100 . Where necessary, build options were specified in packages.yaml, so that the build was reproducible.

After all of the binary artefacts had been built, tests were made of the ability to relocate all of the packages. In particular, the RPATH setting for all libraries and binaries was checked, to ensure that all of the paths were valid in the relocated installation.

To setup a working runtime environment, tests were done of two solutions:

1. Using a Spack view, where a simplified installation directory structure (bin, lib, include, etc.) is created and softlinks to actual locations is used.

2. Setting up a runtime module, which uses a standard environment modules package (Environment Modules or lmod) to manipulate the shell environment to use the new software.

Both solutions proved practical. The advantage of the view is a very compact set of modifications to the environment. However, any additional non-standard environment variables required need to be setup separately, so a wrapper script needs to be provided. The advantage of the modules solution is that all special environment settings are automatically handled by Spack and the module system. However, the modifications to the environment are quite significant (e.g., a very long PATH), which can make certain tasks unwieldy.

The final production solution for runtime and development is still being investigated, with modules favoured for development and views for production running of jobs.

\section{Conclusions and Future Work}

The HSF Packaging Working Group has surveyed many potential solutions to the problem of building, deploying and running large HEP software stacks. From those studies Spack has emerged as a very promising tool. FCC was the first experiment in HEP to demonstrate successfully how Spack can be used and even integrated on top of other, existing solutions. CernVM-FS deployment was demonstrated. SuperNEMO has shown how Spack relieves a small experiment of much of the burden of maintaining a software build and deployment system. The Key4hep project has provided proof-of-concept studies that a complete large HEP software stack can be supported relatively easily, taking advantage of Spack features and the large number of package recipes that are maintained for and by the scientific community.

Work on utilising Spack as the package manager is now continuing, in the context of the Key4hep project and as a replacement for the classic approach to building the LCG stack.

\section{Acknowledgements}

The authors would like to thank the many colleagues active in the HSF Packaging Group, without whom this paper would not have been possible. Special thanks are due to the original convenors of the group, Elizabeth Sexton-Kennedy (FNAL) and Benedikt Hegner (CERN) and to Patrick Gartung (FNAL) and Chris Green (FNAL) who have contributed greatly to the HEP interactions with the Spack core development team. 


\section{References}

[1] HEP software foundation packaging working group, https:// hepsoftwarefoundation.org/workinggroups/packaging.html

[2] T. Gamblin, M. LeGendre, M.R. Collette, G.L. Lee, A. Moody, B.R. de Supinski, S. Futral, The Spack Package Manager: Bringing Order to HPC Software Chaos, in Proceedings of the International Conference for High Performance Computing, Networking, Storage and Analysis (Association for Computing Machinery, New York, NY, USA, 2015), SC '15, ISBN 9781450337236, https : //doi . org/10 . 1145/2807591. 2807623

[3] L. Sexton-Kennedy, B. Hegner, B. Viren, HSF Packaging Working Group Report (2016), https://doi .org/10.5281/zenodo. 1472340

[4] G.A. Stewart, B. Morgan, Use cases for packaging and deployment tools (2020), https://doi.org/10.5281/zenodo. 3634722

[5] M. Benedikt, A. Blondel, O. Brunner, M. Capeans Garrido, F. Cerutti, J. Gutleber, B. Goddard, P. Janot, J.M. Jimenez, M. Klein et al., Tech. Rep. CERN-ACC-2019-0007, CERN, Geneva (2019), https : //cds . cern. ch/record/2653673

[6] J. Cervantes Villanueva, G. Ganis, D. Konstantinov, G. Latyshev, P. Mato Vila, P. Mendez Lorenzo, R. Pacholek, I. Razumov, EPJ Web Conf. 214, 05020 (2019)

[7] J. Blomer, P. Buncic, R. Meusel, G. Ganis, I. Sfiligoi, D. Thain, Computing in Science \& Engineering 17, 61 (2015)

[8] F. Piquemal, Physics of Atomic Nuclei 69, 2096 (2006)

[9] Homebrew, https://brew.sh/ (2020), accessed: 2020-03-06

[10] P. Bambade, T. Barklow, T. Behnke, M. Berggren, J. Brau, P. Burrows, D. Denisov, A. Faus-Golfe, B. Foster, K. Fujii et al., The international linear collider: A global project (2019), 1903.01629

[11] M.J. Boland, U. Felzmann, P.J. Giansiracusa, T.G. Lucas, R.P. Rassool, C. Balazs, T.K. Charles, K. Afanaciev, I. Emeliantchik, A. Ignatenko et al. (CLIC and CLICdp collaborations), Updated baseline for a staged Compact Linear Collider, CERN Yellow Reports: Monographs (CERN, Geneva, 2016), comments: 57 pages, 27 figures, 12 tables, https://cds.cern.ch/record/2210892

[12] T.C.S. Group, CEPC conceptual design report: Volume 1 - accelerator (2018), 1809.00285

[13] Towards a turnkey software stack for HEP experiments, https://indico.cern.ch/ event/773049/contributions/3474763/ (2019) 